\section{PHASE I STUDY OF SAFETY AND ACTIVITY OF PERSONALIZED NEOANTIGEN-BASED VACCINES IN COMBINATION WITH TUMOR TREATING FIELDS FOR NEWLY DIAGNOSED GLIOBLASTOMA PATIENTS}

Julia Kodysh*, Cansu Cimen Bozkus, Mansi Saxena, Marcia Meseck, Alex Rubinsteyn, Tim O'Donnell, Tin Htwe Thin, Rachel Brody, John Mandeli, Nina Bhardwaj, Adilia Hormigo. Icahn School of Medicine at Mount Sinai, New York, NY, USA

Background Glioblastoma (GBM) is one of the most aggressive and lethal cancers (median survival 15 months) characterized by a highly heterogeneous and immunosuppressive tumor microenvironment (TME). New treatments need to include innovative strategies that impact the TME, such as immunomodulation using neoantigen vaccines combined with other modalities like Tumor Treating Fields (TTFields) shown to significantly increase progression-free and overall survival. TTFields disrupt metaphase by delivering $200 \mathrm{kHz}$ low-intensity, alternating electric fields via arrays applied to the scalp. Pre-clinical TTFields studies reveal a role for tumor-infiltrating CD8 + cytotoxic lymphocytes in controlling local disease and decreasing metastases. Therefore, combining the standard care for GBM with a vaccine platform and TTFields may enhance efficacy and improve outcomes.

Methods Adult patients diagnosed with GBM that underwent a maximum debulking surgery, yielding sufficient specimen for sequencing, and treated with standard care (radiotherapy with concurrent and adjuvant Temozolomide chemotherapy) were enrolled to receive personalized neoantigen vaccines with or without TTFields (NCT03223103). Neoantigens were identified using the OpenVax computational pipeline. PBMCs were collected at baseline and weeks 3, 5, 11, 19, 27, 35, 39, 43, 47, 52 and 104. The primary objectives were the safety and toxicity of the vaccines alone and in combination with TTFields. The secondary objectives were patient survival, evaluation of TME using multiplexed immunohistochemistry analysis, and ex vivo and in vitro immunogenicity assays to characterize vaccine-induced epitope-specific $\mathrm{T}$ cell responses.

Results We completed the enrollment of 12 patients (median age 60, range 32-84) between December 2017 and July 2020. The median tumor mutational burden found in the tumors was 3 coding mutations/MB, with a median of 18 predicted neoantigens (range 7 - 155). Each patient was vaccinated with a median of 10 (range 6 -10) synthetic long peptides (each up to 25 amino acids). Patients received up to 14 peptide vaccines, with poly-ICLC as an adjuvant, every other week for two months and once a month thereafter. Some patients elected to receive additional vaccines after the planned 14 injections. The most common adverse events were injection site reactions and flu-like symptoms. Combination with TTFields did not increase toxicity. Nine patients who have a median follow-up of 25 months are alive, with eight remaining disease-free.

Conclusions The combined treatment is well tolerated with minimal addition of adverse events related to vaccination. Preliminary analysis demonstrated induction of robust epitope-specific $\mathrm{T}$ cell responses. More immune monitoring data, TME evaluation, and mature survival analysis are anticipated in November.

Acknowledgements Thank you to all the patients and families who have been so eager to participate in the study.

Ethics Approval This study was approved by the Institutional Review Board of the Icahn School of Medicine at Mount
Sinai, number 17-00204. All the patients gave informed consent.

http://dx.doi.org/10.1136/jitc-2021-SITC2021.334 